\title{
IMPROVED MINIMIZATION METHODS OF PSEUDO KRONECKER EXPRESSIONS FOR MULTIPLE OUTPUT FUNCTIONS
}

\author{
Per Lindgren* \\ Division of Computer Engineering \\ Luleå University of Technology \\ 97187 Luleå, Sweden \\ pln@sm.luth.se
}

\author{
Rolf Drechsler \\ Bernd Becker \\ Institute of Computer Science \\ Albert-Ludwigs-University \\ 79110 Freiburg im Breisgau, Germany \\ \{drechsle/becker\}@informatik.uni-freiburg.de
}

\begin{abstract}
Pseudo Kronecker Expressions (PSDKROs) are a class of AND/EXOR expressions. For a Boolean function with a given variable order the minimal PSDKRO can be derived efficiently using Decision Diagram (DD) techniques. The quality, i.e., the number of products in the expression, of the result is known to be dependent on the variable ordering.

This paper proposes several improvements and enhancements to previous minimization methods. A pruning technique that can be tuned to tradeoff quality for computational resources is presented. By applying dynamic ordering methods, significant improvements to many previously reported results are obtained. Furthermore, a new method for the minimization of multiple output functions is outlined. Experiments on a set of MCNC benchmarks confirm the advantages of the presented algorithms.
\end{abstract}

\section{INTRODUCTION}

The use of EXOR gates in the synthesis process reduces the hardware costs in many cases. Additionally, EXOR based circuits often have nice testability properties. In contrast to AND/OR minimization - that in the meantime is well understood - in AND/EXOR minimization several restricted classes are considered, like Fixed Polarity ReedMuller Expressions (FPRMs) and Kronecker Expressions (KROs). (For an excellent overview see [7].) This subclasses are of interest, since the minimization of general $E x$ clusive Sum of Product Expressions (ESOPs) turned out to be computationally very hard.

As one alternative Pseudo Kronecker Expressions (PSDKROs) [7] have been proposed, since they are an interesting compromise: the resulting 2-level forms are of moderate size and additionally the minimization process can be handled within reasonable time bounds using Multi-Place DDs or more recently Ordered Binary DDs (OBDDs) [2] as shown in [3]. Furthermore, for minimization of totally symmetric functions, the algorithm is shown to derive the exact minima in polynomial time [3].

* The co-operative work was conducted at Albert-Ludwigs-University on a grant from the Swedish Institute.
In this paper we propose a pruning technique for further speeding up the approaches presented in $[7,3]$. The method can be tuned to tradeoff quality for CPU and memory resources, producing heuristic results for previously unmanageable problems. The quality of the minimized PSDKRO expression is known to be dependent on the variable order applied during minimization. We employ dynamic variable ordering strategies and give a comparison of quality and efficiency. Furthermore, we have a closer look at the principles how multiple output functions are handled in presented approaches and outline an alternative method relaxing previous decomposition constraints. This method especially becomes interesting if PSDKRO minimization is seen as a preprocessing for general AND/EXOR minimization or multi-level synthesis. Experimental results are reported that demonstrate the efficiency of the proposed algorithms.

\section{PSEUDO KRONECKER EXPRESSIONS}

In this section we briefly review the essential definitions of Pseudo Kronecker Expressions (PSDKROs). (For more details see $[7,3]$.)

Let $f_{0}\left(f_{1}\right)$ denote the cofactor of $f$ with respect to $\bar{x}(x)$ and $f_{2}$ is defined as $f_{2}=f_{0} \oplus f_{1}, \oplus$ being the Exclusive OR operation. A Boolean function $B^{n} \rightarrow B$ can then be represented by one of the following formulae:

$$
\begin{aligned}
& f=\bar{x} f_{0} \oplus x f_{1} \quad \text { Shannon }(S) \\
& f=f_{0} \oplus x f_{2} \quad \text { positive Davio }(p D) \\
& f=f_{1} \oplus \bar{x} f_{2} \quad \text { negative Davio }(n D)
\end{aligned}
$$

If we apply to a function $f$ either $S, p D$ or $n D$ we get two sub-functions. To each sub-function again $S, p D$ or $n D$ can be applied. This is done until constant functions are reached. If we multiply out the resulting expression we get a 2-level AND/EXOR form, called a PSDKRO. In Section 5 we take a closer look at PSDKRO expressions for multiple output functions.

The decompositions are applied with respect to a fixed variable ordering. Note that the choice of the variable ordering in which the decompositions are applied and the choice 
prod psdkro(node $f$, int prune) \{

1 if $(f==0(1))$ return $0(1)$;

2 if ( $f$.prod defined) return $f . p r o d$;

$3 f_{0}=$ cofactor_o $(f) ; f_{1}=$ cofactor_1 $(f)$;

$4 p_{0}=\operatorname{psdkro}\left(f_{0}\right.$, prune $)$

$5 \quad(P 2) \rightarrow$ if $\left(p_{0} \geq\right.$ prune $)$ return $M A X I N T$;

$6 \quad p_{1}=\operatorname{psdkro}\left(f_{1}\right.$, prune $)$;

$7(P 0, P 1, P 2) \rightarrow$ if $\left(\min \left(p_{0}, p_{1}\right) \geq\right.$ prune $)$ return $M A X I N T$;

$8 f_{2}=\operatorname{EXOR}\left(f_{0}, f_{1}\right)$;

9 if (memory or time limit reached) return $p_{0}+p_{1}$;

$10 p_{2}=\operatorname{psdkro}\left(f_{2}, \operatorname{pf}\left(\right.\right.$ prune, $\left.\left.p_{0}, p_{1}\right)\right)$;

11 f.prod $=p_{0}+p_{1}+p_{2}-\max \left(p_{0}, p_{1}, p_{2}\right)$;

12 return f.prod;

\}

Figure 1: Sketch of the enhanced algorithm

of the decomposition per sub-function largely influences the size of the resulting representation, and may vary from linear to exponential.

Example 1 Consider the parity function $f\left(x_{1}, x_{2}, x_{3}\right)$. A minimal (w.r.t. number of products) SOP form is: $f=$ $\bar{x}_{1} \bar{x}_{2} x_{3}+\bar{x}_{1} x_{2} \bar{x}_{3}+x_{1} \bar{x}_{2} \bar{x}_{3}+x_{1} x_{2} x_{3}$ (4 products, as shown by tracing the 1-paths in Figure $2(a))$, while a minimal PSDKRO form is: $f=x_{1} \oplus x_{2} \oplus x_{3}$ (3 products, as shown by tracing the 1-paths in Figure $2(c))$. For an n-variable parity function the minimal SOP form requires $2^{n-1}$ products, while the minimal PSDKRO requires $n$ products.

\section{PSDKRO MINIMIZATION}

For the implementation of the algorithm we used Ordered Binary Decision Diagrams (OBDDs) [2]. The starting point of our algorithm is the OBDD representation of the function that is to be minimized. A 1-path represents a product term with the cost 1 .

Starting from the root of the OBDD the graph is recursively traversed towards the terminals $\{0,1\}$. At each node an EXOR operation is carried out, which by the use of OBDDs can be performed in polynomial time [2]. From the fact that for each of the decomposition formulae from Section 2 only two out of the three possible successors $f_{0}, f_{1}$ and $f_{2}$ are needed to represent the function, we can choose the two least costly in order to minimize the resulting PSDKRO ${ }^{1}$.

For each node $f$ the minimal number of product terms needed for the representation as a PSDKRO is stored in the variable f.prod. Thus, each node has to be evaluated only once.

Example 2 Consider the algorithm in Figure I (without the "prune" related code). Assume that $p_{0}=2, p_{1}=2$ and

\footnotetext{
${ }^{1}$ The basic idea of the algorithm is the same as used in [7] for PSDKRO minimization.
}

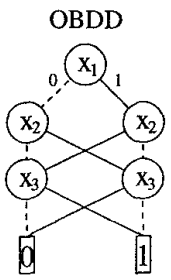

(a)

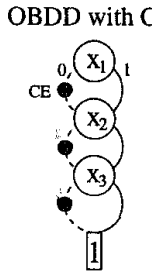

(b)

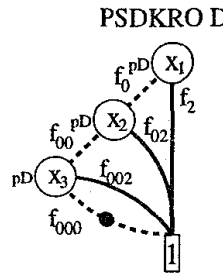

(c)
Figure 2: Parity function

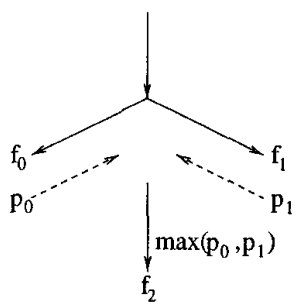

(a)

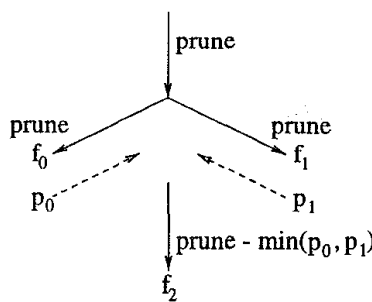

(b)
Figure 3: Pruning of search space

$p_{2}=1$ (lines 4, 6, and 10). The cost of $S, p D$ and $n D$ decompositions are $p_{0}+p_{1}=4, p_{0}+p_{2}=3$ and $p_{1}+$ $p_{2}=3$ accordingly. Line 11 computes the least cost 3 . Figure 2 (c) shows a PSDKRO DD for the parity function $f\left(x_{1}, x_{2}, x_{3}\right)$ using only $p D$ decompositions. This example relates to the decomposition for $x_{1}$.

\subsection{An Enhanced Minimization Algorithm}

Previous algorithms exhaustively traverse the search space to find optimal decompositions, $[7,3]$. We make the following critical observations: The OBDD representation holds an initial (non-optimal) PSDKRO solution having only Shannon decompositions, $\left(\bar{x} f_{0} \oplus x f_{1}\right)$ with the cost $p_{0}+p_{1} . f_{2}$ is a part of the solution iff $p_{2}<\max \left(p_{0}, p_{1}\right)$, i.e., $f_{2}$ must be less costly than at least one other cofactor $f_{0}$ or $f_{1}$ (see Figure $3\left(\right.$ a)). Given a cost limit "prune", $f_{2}$ is a part of the solution iff $p_{2}<$ prune - $\min \left(p_{0}, p_{1}\right)$, since the cost of a Davio decomposition is $\min \left(p_{0}, p_{1}\right)+p_{2}$, which in turn must be less than "prune" (see Figure 3 (b)). Our hands on experiences show that a high cost for $f_{0}$ or $f_{1}$ in many cases also leads to a high cost for $f_{2}$.

These observations are exploited in Figure 1, lines 5, 7, 9, 10 and the additional pruning parameter. In the following three pruning methods are considered.

PO By returning MAXINT whenever the cost exceeds the cost limit, further "pointless" traversal is prevented, line 7. The pruning function "pf", line 10 , chooses the minimum of (a) and (b) as the cost limit for $f_{2}$, i.e., $\min \left(\max \left(p_{0}, p_{1}\right)\right.$, prune $\left.-\min \left(p_{0}, p_{1}\right)\right)$. 
PI By the choice of a more aggressive pruning function the algorithm can be tuned to tradeoff quality for computational resources. By choosing $\min \left(p_{0}, p_{1}\right)$ for condition (a), computation of $f_{2}$ is aborted as soon as $p_{2}$ equals either $p_{0}$ or $p_{1}$. This realizes the idea to traverse $f_{2}$ only where Davio decomposition is clearly favorable.

$P 2$ Intrigued by the last observation, we sought an answer whether it might be sufficient to inspect only one of the cofactors to estimate the cost. This question is manifested in line 5 , which aborts traversal whenever $p_{0}$ reaches the cost limit. In the following $P 2$, is used with the exact pruning function from $P O$.

Furthermore as a last effort to reduce the complexity, line 9 computes the cost of a Shannon decomposition, (which is inherent in the OBDD representation as observed above), whenever computational resources are exhausted.

The motivation to apply pruning and heuristic approaches is to save CPU and memory resources, thus enable us to tackle problems for which previous attempts fail due to time or memory limits.

\subsection{Literal Minimization}

Simultaneous minimization of literal count is straightforward. Whenever we can choose between decomposition types, (all producing the minimal number of products), we choose the decomposition producing the least number of literals, i.e., f.lit $=\min \left(l_{S}, l_{p D}, l_{n D}\right)$ corresponding to the literal count of $\mathrm{S}, \mathrm{pD}$ and $\mathrm{nD}$ decompositions, respectively. Further implementation details are left out because of page limitations.

\subsection{Complennented Edges}

The use of Complemented Edges (CEs) in OBDD packages [1] has several advantages; firstly the number of nodes needed to represent the function is often reduced due to increased overlapping, and secondly further controlling cases for computation can be utilized. The presented algorithm depends heavily on the efficiency of EXOR operations. By the use of complemented edges the result of $f_{0} \oplus f_{1}$ can be directly derived whenever $f_{0}=\bar{f}_{1}$. In Figure 2 all EXOR computations leading to the minimized PSDKRO (c), can be derived in constant time using CEs (b).

\section{INFLUENCE OF VARIABLE ORDERING}

In [7] the effect of variable ordering was concluded by enumerating all possible orderings for a given function and minimizing the corresponding PSDKROs. However, no method to approach the ordering problem was reported. In general dynamic variable ordering [6] has proven useful to many DD problems and has been applied in [4] to PSDKRO based Maitra term minimization. In this paper we adopt dynamic variable ordering directly to the problem of PSDKRO minimization and present two different approaches.
R1 Move to top strategy. First we compute the cost using the initial ordering of the input variables, e.g., $\left(x_{1}, x_{2}, x_{3}, . ., x_{n}\right)$, where $x_{1}$ is the top variable. Then we try all other variables as the top variable and store the best ordering obtained, e.g., $\left(x_{3}, x_{1}, x_{2}, . ., x_{n}\right)$. We now repeat the procedure, e.g., with $x_{3}$ as the new initial top variable, until no further optimization is obtained or a time limit is reached.

R2 Local variable exchange method. Basically the "sifting" algorithm presented in [6]. Unfortunately the cost cannot be derived from local operations, complete PSDKRO minimization is required for each variable order applied.

\section{MULTIPLE OUTPUT FUNCTIONS}

In [7] the algorithm for minimizing the PSDKRO for Multiple Output (MO) functions is based on Multi-Place DDs, for which each terminal corresponds to an output vector. However, as used e.g., in [8], the terminals can be encoded by a set of Output Selection (OS) variables, (one variablenode for each output function). This encoding gives us the freedom to change the grouping of output functions by the position of the OS nodes. Moving all OS nodes to the top of the DD allows applying decompositions with respect to each output function separately, while keeping the OS nodes at the bottom of the DD corresponds to the representation used in [7]. In the latter case, decompositions are applied simultaneously to all output functions (analogous to a multi-valued interpretation). Besides these two extremes, OS nodes can be interleaved with the DD nodes. The introduction of output selection variables increases the number of 1-paths (i.e., the cost function), in turn leading to an overestimation of the number of required products. To correctly account for product sharing of MO functions is to the best of our knowledge trivial only when the OS nodes is at the bottom of the diagram.

\section{EXPERIMENTAL RESULTS}

In this section we present experimental results for a set of MCNC PLA benchmark functions performed on a Sun $\mathrm{Ul}$ $\mathrm{tra} l$ workstation with $256 \mathrm{Mb}$ RAM. (All totally symmetric functions are marked by *.) For the implementation we used the CUDD 2.1.2 BDD-package [9]. The peak memory requirement of the performed experiments was measured to $75 \mathrm{Mb}$. Dynamic reordering methods are implemented by synthesis operations, as internal BDD cost functions do not apply.

In a first set of experiments (see Table 1) we show the effect of pruning and heuristic minimization under the variable ordering given by the PLAs. The results obtained by re-implementing the algorithm for MO PSDKRO minimization from [3] are marked $M O$. Columns $P O$ and $P I$ show the results for the exact and aggressive pruning functions, 


\begin{tabular}{|l|r|r|r|r|r|r|r|r|}
\hline name & in/out & \multicolumn{4}{|c|}{ Number of Products } & \multicolumn{4}{|c|}{ CPU Time in Seconds } \\
& & $M O / P O$ & $P 1$ & $P 2$ & $M O$ & $P O$ & $P I$ & $P 2$ \\
\hline 5xp1 & $7 / 10$ & 47 & 51 & 47 & 0.01 & 0.01 & 0.01 & 0.01 \\
add6 & $12 / 7$ & 132 & 133 & 132 & 0.03 & 0.03 & 0.03 & 0.03 \\
bc0 & $26 / 11$ & 180 & 221 & 180 & 5.34 & 2.79 & 0.81 & 1.34 \\
co14* & $14^{\prime} 1$ & 14 & 14 & 14 & 0.01 & 0.01 & 0.01 & 0.01 \\
duke2 & $22 / 29$ & 108 & 157 & 108 & 25.49 & 23.88 & 9.19 & 17.09 \\
in2 & $19 / 10$ & 117 & 128 & 117 & 2.59 & 2.49 & 1.13 & 2.00 \\
in7 & $26 / 10$ & 42 & 82 & 42 & 0.17 & 0.17 & 0.16 & 0.16 \\
inc & $7 / 9$ & 31 & 33 & 31 & 0.01 & 0.01 & 0.01 & 0.01 \\
intb & $15 / 7$ & 500 & 1153 & 500 & 0.75 & 0.72 & 0.59 & 0.69 \\
misex3 & $14 / 14$ & 754 & 1199 & 754 & 5.11 & 4.29 & 2.92 & 3.94 \\
rd53* & $5 / 3$ & 20 & 27 & 20 & 0.01 & 0.01 & 0.01 & 0.01 \\
rd73* & $7 / 3$ & 63 & 79 & 63 & 0.01 & 0.01 & 0.01 & 0.01 \\
rd84* & $8 / 4$ & 107 & 134 & 107 & 0.01 & 0.01 & 0.01 & 0.01 \\
sao2 & $10 / 4$ & 41 & 63 & 41 & 0.01 & 0.01 & 0.01 & 0.01 \\
t481 & $16 / 1$ & 13 & 17 & 13 & 0.01 & 0.01 & 0.01 & 0.01 \\
tial & $14 / 8$ & 939 & 1231 & 939 & 1.30 & 1.27 & 1.21 & 1.18 \\
vg2 & $25 / 8$ & 293 & 401 & 295 & 1.54 & 1.54 & 1.45 & 1.38 \\
x6dn & $39 / 5$ & 104 & 161 & 104 & 5.90 & 1.68 & 0.44 & 0.38 \\
\hline
\end{tabular}

Table 1: Pruning experiments

respectively. The latter is clearly more effickent while compromising the quality. Under the assumption that inspecting a single cofactor is enough for cost estimation $P 2$ efficiently obtains optimal results for the given set of benchmarks except for the near optimal result for $\mathrm{vg} 2$.

In the second set of experiments the results for different variable reordering methods are compared (see Table 2). Columns $R I$ and $R 2$ show the effect of dynamic reordering methods applied to the $M O$ minimization method. To speed up the cost calculation heuristic $P 2$ is used. In general better results are obtained from the time consuming sifting method $R 2$. The results show significant improvements to many previously reported results $M O$ in Table 1 . Columns $S O$ show the results from Single Output (SO) minimization, (corresponding to OS nodes at top, Section 5), using the PLA variable orderings. During the minimization the number of products required is overestimated, thus leading to nonoptimal solutions. However, common products are counted only once in the reported results. Compared to $M O$ in Table 1 , SO minimization shows quality improvements in many cases. For some benchmarks (e.g., intb, tial and vg2) SO even compares to results obtained by variable reordering. Furthermore, for the benchmark set SO minimization consumes significantly less computational resources than MO minimization.

Based on these promising results it is focus of current work to integrate the PSDKRO minimizer in an EXOR based synthesis tool. Another open problem is the optimization of incompletely specified functions. First results can be found in [5].

\section{Acknowledgment}

The authors wish to thank Prof. T. Sasao for inspiring discussions on the PSDKRO minimization problem.

\begin{tabular}{|l|r|r|r|r|r|r|r|}
\hline name & in/out & \multicolumn{1}{|c|}{ Number of Products } & \multicolumn{3}{|c|}{ CPU Time in Seconds } \\
& & $R I$ & $R 2$ & $S O$ & $R I$ & $R 2$ & $S O$ \\
\hline 5xp1 & $7 / 10$ & 43 & 42 & 51 & 0.05 & 0.13 & 0.01 \\
add6 & $12 / 7$ & 132 & 132 & 132 & 0.32 & 1.97 & 0.03 \\
bc0 & $26 / 11$ & 175 & 174 & 299 & 43.93 & 289.58 & 0.51 \\
co14* & $14 / 1$ & - & - & 14 & - & - & 0.01 \\
duke2 & $22 / 29$ & 91 & 85 & 153 & 920.43 & 3949.75 & 0.08 \\
in2 & $19 / 10$ & 117 & 117 & 138 & 18.83 & 139.98 & 0.09 \\
in7 & $26 / 10$ & 36 & 37 & 51 & 2.06 & 34.66 & 0.02 \\
inc & $7 / 9$ & 31 & 30. & 47 & 0.03 & 0.11 & 0.00 \\
intb & $15 / 7$ & 402 & 401 & 390 & 33.98 & 62.89 & 0.21 \\
misex3 & $14 / 14$ & 686 & 638 & 929 & 71.89 & 478.56 & 0.39 \\
rd53* & $5 / 3$ & - & - & 20 & - & - & 0.01 \\
rd73* & $7 / 3$ & - & - & 55 & - & - & 0.01 \\
rd84* & $8 / 4$ & - & - & 90 & - & - & 0.01 \\
sao2 & $10 / 4$ & 33 & 33 & 48 & 0.10 & 0.30 & 0.01 \\
t481 & $16 / 1$ & 13 & 13 & 13 & 0.02 & 0.25 & 0.01 \\
tial & $14 / 8$ & 728 & 820 & 752 & 39.99 & 104.72 & 0.36 \\
vg2 & $25 / 8$ & 250 & 216 & 233 & 75.25 & 324.79 & 0.21 \\
x6dn & $39 / 5$ & 101 & 98 & 118 & 29.61 & 196.35 & 0.14 \\
\hline
\end{tabular}

Table 2: Ordering experiments

\section{REFERENCES}

[1] K.S. Brace, R.L. Rudell, and R.E. Bryant. Efficient implementation of a BDD package. In Design Automation Conf., pages 40-45, 1990.

[2] R.E. Bryant. Graph - based algorithms for Boolean function manipulation. IEEE Trans. on Comp., 35(8):677-691, 1986.

[3] R. Drechsler. Pseudo Kronecker expressions for symmetric functions. In VLSI Design Conf., pages 511-513, 1997.

[4] G. Lee. Logic synthesis for cellular architecture FPGAs using BDDs. In ASP Design Automation Conf., pages 253-258, 1997.

[5] G. Lee and R. Drechsler. ETDD-based generation of complex terms for incompletely specified boolean functions. In ASP Design Automation Conf., 1998.

[6] R. Rudell. Dynamic variable ordering for ordered binary decision diagrams. In Int'l Conf. on $C A D$, pages 42-47, 1993.

[7] T. Sasao. AND-EXOR expressions and their optimization. In T. Sasao, editor, Logic Synthesis and Optimization, pages 287-312. Kluwer Academic Publisher, 1993.

[8] T. Sasao and J.T. Butler. A method to represent multiple-output switching functions by using multivalued decision diagrams. In Int'l Symp. on multivalued Logic, pages 248-254, 1996.

[9] F. Somenzi. CUDD: CU Decision Diagram Package Release 2.1.2. University of Colorado at Boulder, 1997. 